\title{
Approximate Analytical Description for Fundamental-Mode Fields of Graded-Index Fibers: Beyond the Gaussian Approximation
}

\author{
Qing Cao and Sien Chi, Fellow, OSA
}

\begin{abstract}
An approximate analytical description for fundamental-mode fields of graded-index fibers is explicitly presented by use of the power-series expansion method, the maximum-value condition at the fiber axis, the decay properties of fundamental-mode fields at large distance from the fiber axis, and the approximate modal parameters $U$ obtained from the Gaussian approximation. This analytical description is much more accurate than the Gaussian approximation and at the same time keep the simplicity of the latter. As two special examples, we present the approximate analytical formulas for the fundamental-mode fields of a step profile fiber and a Gaussian profile fiber, and we find that they are both highly accurate in the single-mode range by comparing them with the corresponding exact solutions.
\end{abstract}

Index Terms-Fundamental mode, optical waveguide, power-series expansion, single-mode fiber.

\section{INTRODUCTION}

$\mathbf{T}$ HERE is much interest in the determination of the modal fields and the propagation constants of fundamental modes of graded-index fibers due to the great progress of single-mode fiber communication systems. However, exact analytical solutions are possible only for a limited class of index profiles, such as the step profile [1], [2], the clad parabolic profile [3], the power-law profiles [4], [5], and the infinite parabolic profile [6]. Even for these special profiles (except for the unphysical infinite parabolic profile [6]), the solutions [1]-[5] are still given in special functions or in the sum of many power series. In almost all cases, one has to use either approximate or numerical methods. At present, it is possible to use numerical methods to obtain high accuracy, but one can not obtain much physical insight as with the analytical expressions presented by approximate methods. Among the approximate methods, the simple Gaussian approximation [7] is good for the fundamental modes of graded-index fibers with large $V$ value (i.e., $V$ is near or larger than the cutoff frequency $V_{c}$ ). However, when the normalized frequency $V$ gets smaller, the Gaussian approximation

\footnotetext{
Manuscript received February 14, 2000; revised August 10, 2000. This work was supported by National Science Council of China under Contract NSC 88-2215-E-009-006. The work of Q. Cao was done while with the Institute of Electro-Optical Engineering, National Chiao Tung University and also with the Shanghai Institute of Optics and Fine Mechanics.

Q. Cao was with the Institute of Electro-Optical Engineering, National Chiao Tung University, Hsinchu, Taiwan 300, China, and also was with the Shanghai Institute of Optics and Fine Mechanics, Shanghai 201800, China. He is now with the Institut d'Optique, CNRS, B.P. 147, Orsay Cedex F-91403, France.

S. Chi is with the Institute of Electro-Optical Engineering, National Chiao

Tung University, Hsinchu, Taiwan 300, China.

Publisher Item Identifier S 0733-8724(01)03557-5.
}

for the fundamental-mode fields becomes less accurate. In addition, the Gaussian approximation can not correctly describe the fundamental-mode fields at large distance from the fiber axes. To improve the accuracy, many people have presented various modified versions, such as the Gaussian-exponential [8], the Gaussian-Hankel [9], the generalized Gaussian [10], the extended Gaussian [11], and the Laguerre-Gauss/Bessel expansion approximations [12]. These modified versions are far more accurate than the simple Gaussian approximation. However, the optimizing processes of these modified variational methods are much more complicated than that of the Gaussian approximation.

Unlike the high-order modes, the fundamental mode of a graded-index fiber has some special properties, such as the maximum-value point is always located at the fiber axis, the modal field is always larger than zero in the whole range of $0 \leq R<\infty$. As we show below, these special properties allow us to establish a simple power-series method to obtain the approximate fundamental-mode field when the modal parameter $U$ is known. Fortunately, for those typical graded-index fibers, such as the power law profile fibers [4], [5], [7], [13] and the Gaussian profile fiber [13]-[16], the Gaussian approximation can still present highly accurate modal parameters $U$ in the single-mode range ([7, Fig. 14] and [13, Fig. 15-1], note that the normalized frequency $V$ of the practical single-mode fibers are usually in the range of $1.5 \leq V \leq 3.0$ ), though in this case the Gaussian approximation fails to provide the accurate fundamental-mode fields.

In this paper, we shall use the power-series expansion method, the maximum-value condition at the fiber axes, the positive-value property of the fundamental-mode fields, the decay property of the fundamental-mode fields at large distance from the fiber axes, and the modal parameters $U$ determined by the Gaussian approximation [7] to present a approximate analytical description for the fundamental-mode fields of graded-index fibers. In particular, as two special examples, we shall provide highly accurate closed-form formulas for the fundamental-mode fields of a step profile fiber and a Gaussian profile fiber.

\section{Physical Considerations And Mathematical TREATMENTS}

Let us consider a graded-index fiber whose radial index distribution $n(R)$ has the form

$$
n^{2}(R)=n_{c o}^{2}[1-2 \Delta f(R)]
$$


where

$$
\begin{array}{ll}
n_{c o} & \text { maximum index at the fiber axis; } \\
R=r / \rho & \begin{array}{l}
\text { normalized radial coordinate; } \\
\text { characteristic radius of the fiber core; }
\end{array} \\
\rho & \text { radial coordinate; } \\
r & \text { total variation in the profile, } \Delta \ll 1 ;
\end{array}
$$

$f(R) \quad$ profile function and satisfies the relations $f(0)=$ 0 and $f(\infty)=1$.

It is well known that the modal field $\phi(r)$ and the propagation constant $\beta$ of the fundamental mode of a weakly guiding fiber satisfy the following eigen equation:[13]

$$
\frac{d^{2} \phi}{d r^{2}}+\frac{1}{r} \frac{d \phi}{d r}+\left[n^{2} k^{2}-\beta^{2}\right] \phi=0
$$

where $k=2 \pi / \lambda$, is the wavenumber in free space, $\lambda$ is the wavelength in free space.

Substituting (1) into (2), and using the normalized radial coordinate $R=r / \rho$ to replace the radial coordinate $r$, one can obtain

$$
\frac{d^{2} \phi}{d R^{2}}+\frac{1}{R} \frac{d \phi}{d R}+U^{2} \phi-V^{2} f(R) \phi=0
$$

where $V=k \rho n_{c o} \sqrt{2 \Delta}$, is the normalized frequency, $U=$ $\rho \sqrt{n_{c o}^{2} k^{2}-\beta^{2}}$, is the dimensionless modal parameter for fiber core.

Similarly to the evanescent field expression of the WKB method [17], we first express the modal field $\phi(R)$ as the following form

$$
\phi(R)=A \exp \left[-\int_{0}^{R} P(R) d R\right]
$$

where $A$ is a normalization constant, $P(R)$ is a real function of the variable $R$. Apparently, this expression is consistent with the real physical phenomenon that the fundamental-mode field of a graded-index fiber, such as a power law profile fiber, is always larger than zero in the whole range of $0 \leq R<\infty$. Unlike the WKB method [17], we do not assume the triangular-function soultion because the fundamental-mode field has no node in the whole range of $0 \leq R<\infty$ at all.

Substituting (4) into (3), one can obtain

$$
-P^{\prime}+P^{2}-\frac{P}{R}=-U^{2}+V^{2} f(R)
$$

One may notice that [18] provided a similar treatment for the eigen equation of single-mode fibers. Especially, one can prove that (5) here is completely equivalently to (6) of [18] by taking the relation $P(R)=-G(R)$ into account. This relation shows that our work is somewhat related to [18]. However, it is necessary to point out that they are apparently different. Reference [18] is mainly on the numerical algorithm, but our work is mainly on the approximately analytical description.

From the point of view of physics, a real fiber should have a uniform-index cladding. It is well known that the fundamental-mode field $\phi(R)$ in the uniform-index cladding can be analytically expressed as $\phi(R) \propto K_{0}(W R)$, where $K_{0}(u)$ is the modified Bessel function of the zeroth order and $W$ is given by $W=\sqrt{V^{2}-U^{2}}$. In terms of this relation, (4) and the assumption that the core-cladding interface is located at the $R=1$ point for convenience, one can obtain the boundary condition $P(1)=-G(1)=W K_{1}(W) / K_{0}(W)$, where $K_{1}(W)$ is the modified Bessel function of the first order. Obviously, this boundary condition is just [18, eq. (8b)]. In order to reduce the computation, [18] employed this boundary. However, we shall not use this boundary condition in the remainder of this paper. This is mainly due to the following two considerations. 1) We shall try to use some elementary function to approximately analytically describe the fundamental-mode field of single-mode fiber because of their simplicity. However, the modified Bessel functions appeared in the above-mentioned boundary condition are not elementary function. 2) Smooth profile functions such as the Gaussian profile function are very useful, even though, strictly speaking, those fibers with such smooth profile functions do not exist. However, for those smooth profile fibers, the boundary condition $P(1)=W K_{1}(W) / K_{0}(W)$ becomes invalid.

By use of (4) and the maximum-value condition $\phi^{\prime}(0)=$ $-P(0) \phi(0)=0$ at the $R=0$ point, one can prove that $P(0)=$ 0 at the $R=0$ point. We then expand $P(R)$ and the profile function $f(R)$ (in this paper, we only consider those gradedindex fibers whose profile function $f(R)$ can be expanded as the power series for small $R$ ) as the power-series form

$$
\begin{array}{r}
P=\sum_{m=1}^{m_{0}} a_{m} R^{m}+O\left(R^{m_{0}+1}\right) \\
f(R)=\sum_{m=1}^{m_{0}-1} b_{m} R^{m}+O\left(R^{m_{0}}\right)
\end{array}
$$

in the region near the fiber axis, where we have used the properties that $P(0)=0$ and $f(0)=0$. One may notice that the power series of (6) and the power series of (7) are independent of each other, and therefore one can use two independent numbers to express the powers of their highest-order terms that are taken into account, respectively. However, we use two related numbers $m_{0}$ and $m_{0}-1$ to express the powers of the highest-order terms of (6) and (7), respectively, because we hope the highest-order terms of $P^{\prime}$ has the same powers as that of $f(R)$ in (5).

The high-order terms whose powers are higher than $m_{0}$ in the function $P(R)$ and the high-order terms whose powers are higher than $m_{0}-1$ in the profile function $f(R)$ have been ignored. Obviously, the expansion coefficients $b_{m}$ can be determined by $b_{m}=f^{(m)}(0) / m$ !, where $f^{(m)}(0)$ is the $m$ th-order derivative of $f(R)$ at the $R=0$ point. The number $m_{0}$ of the terms of the power series for the function $P(R)$ can be determined according to practical needs. Generally speaking, the larger the number $m_{0}$ is, the better the obtained result is, but the more complicated the expression for the function $P(R)$ is. For the fundamental-mode field of an ordinary graded-index fiber, the first several terms are sufficient. In the examples of Section III, we shall use only the first five terms (excluding the terms whose coefficients $a_{m}$ are equal to zero). 
Substituting (6) and (7) into (5), and ignoring the high-order terms whose powers are higher than $m_{0}-1$, one can obtain the following recurrence relations:

$$
\begin{aligned}
a_{1} & =\frac{U^{2}}{2} \\
a_{2} & =-\frac{V^{2} b_{1}}{3} \\
a_{m} & =\frac{1}{m+1}\left(\sum_{j=1}^{m-2} a_{j} a_{m-j-1}-V^{2} b_{m-1}\right) \\
& \quad 3 \leq m \leq m_{0} .
\end{aligned}
$$

It is necessary to point out that these solutions are dependent on the modal parameter $U$. In almost all cases, the exact $U$ values cannot be given in closed form. Fortunately, the simple Gaussian approximation can still provide highly accurate expressions for the $U$ values in the single-mode range [7], [13] (as low as $V=1.0$ ). Therefore, we suggest to directly substitute the $U$ values that are obtained from the Gaussian approximation into (8)-(10) to approximately determine the concrete values of the parameters $a_{m}$. It is worth mentioning that the power-series expansion method presented here is completely different from the previous power-series soultions for the fundamental modes of graded-index fibers [4], [5], [19]. The latter directly expand the fundamental-mode field $\phi(R)$ as power-series and therefore has slow convergence property, but we now expand the function $P(R)$ as power series and obtain a fast convergent solution. In fact, as we state above, in our expansion method, the first several terms are usually sufficient for the function $P(R)$.

Let us now investigate the asymptotic behavior of the fundamental-mode field of a graded-index fiber at large $R$. Similarly to the WKB approximation [17], from (5) one can deduce that $P(R)=W=\sqrt{V^{2}-U^{2}}$ and $P^{\prime}(R)=0$ when $R \rightarrow \infty$, where $W$ is the modal parameter for the fiber cladding. Therefore, it is reasonable to use $P_{0}(R)=W$ as the zero-order approximation at large $R$, and then employ the perturbation method to obtain the first-order correction term $P_{1}(R)$. Similarly to the WKB method [17], in terms of (5) one can easily establish the following perturbation equation:

$$
2 P_{0} P_{1}-\frac{P_{0}}{R}=V^{2}[f(R)-1]
$$

for large $R$, where the terms $P_{1}^{2}(R), P_{1}^{\prime}(R)$ and $P_{1} / R$ have been ignored. From (11), one can obtain

$$
P \approx P_{0}+P_{1} \approx W+\frac{1}{2 R}
$$

where we have ignored the term $V^{2}[f(R)-1] /(2 W)$ because it is usually far smaller than the two terms of the right-hand side of
(12) for large $R$. For example, for a power law profile fiber, the term $V^{2}[f(R)-1] /(2 W)$ is exactly equal to zero in the range of $R \geq 1$.

We then use the following method to joint the two kinds of solutions that are given by (6) and (12), respectively. When $R \leq$ $R_{0}$, the function $P(R)$ is given by (6), and when $R>R_{0}$, the function $P(R)$ is given by (12), where the joint point $R_{0}$ is determined by the equation

$$
\sum_{m=1}^{m_{0}} a_{m} R_{0}^{m}=W+\frac{1}{2 R_{0}} .
$$

One can easily prove that (13) can ensure that the fundamental-mode field $\phi(R)$ and its first-order derivative $\phi^{\prime}(R)$ are both continuous at the joint point $R=R_{0}$. One may want to know whether or not the joint point $R_{0}$ used here is related to the so called turning point $R_{t}$ used in the WKB method [17]. We point out that they are completely different. The former is defined by (13), but the latter is determined by the relation $n^{2}\left(R_{t}\right) k^{2}-\beta^{2}=0$ [17]. As we point out before, the fundamental-mode field $\phi(R)$ and its first-order derivative $\phi^{\prime}(R)$ are both continuous at the joint point $R_{0}$; but according to the WKB method, the modal field is divergent at the turning point $R_{t}$. In addition, we also want to emphasize the well-known result that the WKB method is invalid for the fundamental-mode field. Therefore, we do not discuss the turning point problem in this paper. In practical applications, (13) can be easily solved by numerical approach.

After obtaining the coefficients $a_{m}$ and the joint point $R_{0}$, one can analytically express the fundamental-mode field $\phi(R)$ as the closed form (14) shown at the bottom of the page.

One can obtain a more accurate $U$ value than that of the Gaussian approximation if one substitute the solution of (14) into the expression

$$
\begin{aligned}
U^{2} & =\frac{\int_{0}^{\infty}\left[(d \phi / d R)^{2}+V^{2} f(R) \phi^{2}\right] R d R}{\int_{0}^{\infty} \phi^{2} R d R} \\
& =\frac{\int_{0}^{\infty}\left[P^{2}+V^{2} f(R)\right] \phi^{2} R d R}{\int_{0}^{\infty} \phi^{2} R d R}
\end{aligned}
$$

which can be derived from (3) and (4), because the solution given by (14) is more accurate than the corresponding Gaussian approximation solution. In order to understand this statement better, we shall present two concrete numerical results in Sections III-A and -B.

$$
\phi(R)=\left\{\begin{array}{ll}
A \exp \left[-\sum_{m=1}^{m_{0}} a_{m} R^{m+1} /(m+1)\right], & 0 \leq R \leq R_{0}, \\
A \sqrt{R_{0} / R} \exp \left[-W\left(R-R_{0}\right)-\sum_{m=1}^{m_{0}} a_{m} R_{0}^{m+1} /(m+1)\right], & R \geq R_{0} .
\end{array} .\right.
$$


It is interesting to explain why the fundamental-mode field of a graded-index fiber has an approximate Gaussian distribution when the normalized frequency $V$ becomes large. Our theoretical treatments imply a good explanation. If one let $m_{0}=$ 1 , then one can obtain the zero-order approximation $\phi(R)=$ $A \exp \left(-U^{2} R^{2} / 4\right)$, which is a Gaussian distribution. One may notice the interesting result that the width of this Gaussian distribution is only (directly) related to the modal parameter $U$. Usually, when the normalized frequency $V$ becomes large, the terms $a_{m} R^{m+1} /(m+1)(m \geq 2)$ become negligible with the first term $a_{1} R^{2} / 2=U^{2} R^{2} / 4$ before the first term $U^{2} R^{2} / 4$ has become rather large. As a consequence, in this case, the zero-order approximation is a good one in the region where the modal field is remarkably different from zero. This is why the fundamental-mode field of a graded-index fiber shows a nearly Gaussian distribution when $V$ is large.

\section{EXAMPLES}

To understand the above analytical description better, let us now present two special examples. One is the fundamental-mode field of a step profile fiber, the other is the fundamental-mode field of a Gaussian profile fiber. These two kinds of graded-index fibers are both typical. Therefore, the investigation on their fundamantal-mode fields has practical meanings.

\section{A. The Step Profile Fiber}

For a step profile fiber whose profile function is given by $f(R)=0$ for $R \leq 1$ and $f(R)=1$ for $R>1$, the expansion coefficients $b_{m}$ are determined to be $b_{m}=0$ for all orders. Substituting these relations into (8)-(10) and letting $m_{0}=9$, one can obtain $a_{2}=a_{4}=a_{6}=a_{8}=0, a_{1}=U^{2} / 2$, $a_{3}=U^{4} / 16, a_{5}=U^{6} / 96, a_{7}=11 U^{8} / 6144$ and $a_{9}=$ $19 U^{10} / 61440$, where the modal parameter $U$ is approximately given by $U^{2}=1+2 \ln V$ according to the Gaussian approximation [13], [14]. Substituting the coefficients $a_{m}$ and the relations $U^{2}=1+2 \ln V$ and $W=\sqrt{V^{2}-1-2 \ln V}$ into (13), one can determine the joint point $R_{0}$. The change of $R_{0}$ with the variable $V$ is shown in Fig. 1. From Fig. 1 one can see that $R_{0}$ is nearly invariant in the range of $1 \leq V \leq 4$. To more conveniently use the approximate analytical formulas of (14), we now use the approximate expression

$$
R_{0}= \begin{cases}-0.0634 V^{2}+0.2489 V+0.7553, & 1 \leq V \leq 2 \\ 1.0, & 2<V \leq 4\end{cases}
$$

to fit the function $R_{0}(V)$ in the single-mode range. From (16) one can find that $R_{0}(V)$ is a approximate parabolic function in the range of $1 \leq V \leq 2$ and is approximately equal to 1.0 in the range of $2<V \leq 4$. From Fig. 1 one can further find that the total change of $R_{0}$ in the range of $1 \leq V \leq 4$ is very small and the central point is about $R_{0}=0.97$.

The error of (16) with the exact values is less than $0.34 \%$ in the range of $1 \leq V \leq 2$ and less than $0.52 \%$ in the range of $2<V \leq 4$. The modal field distribution is not sensitive to this

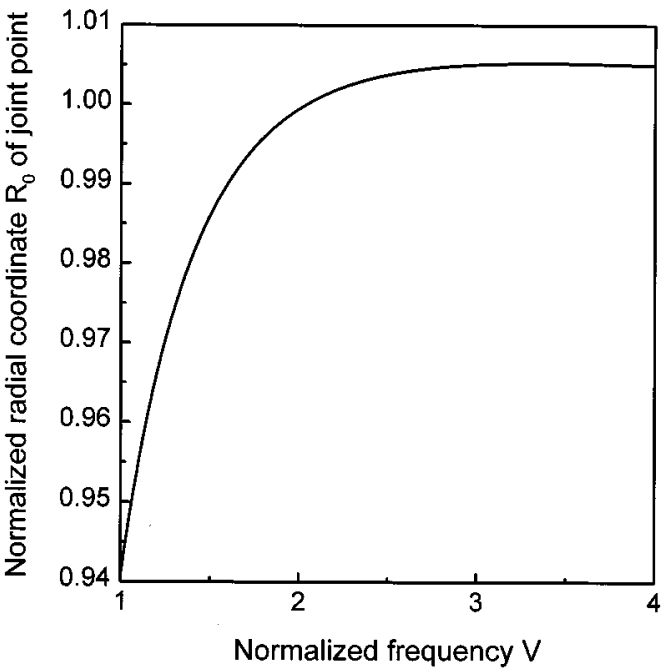

Fig. 1. Functional relation between the normalized radial coordinate $R_{0}(V)$ of the joint point and the normalized frequency $V$ : The step profile fiber.

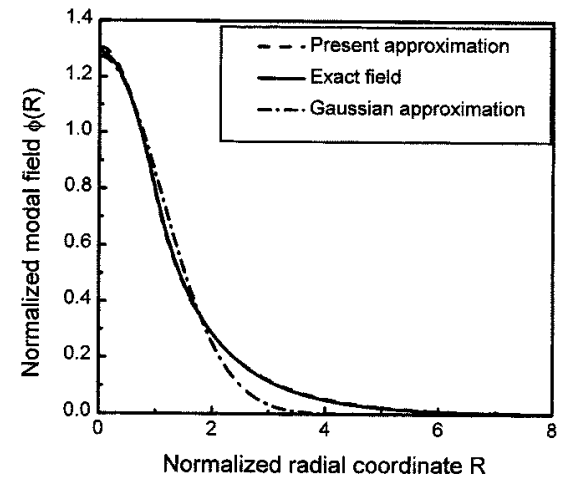

(a)

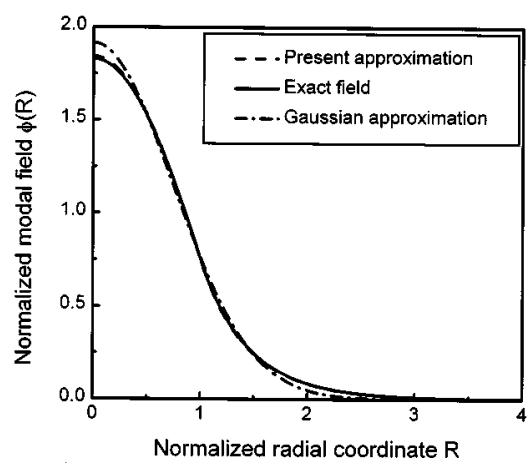

(b)

Fig. 2. Comparison among our approximate formula, the Gaussian approximation and the exact solution: The step profile fiber: (a) $V=1.5$ and (b) $V=2.5$.

small error, because (6) and (12) are both approximately valid in the region near the $R=R_{0}$ point. In practical applications, one can directly insert (16) and the expressions of $a_{m}, U$ and $W$ (they are all explicitly given as the functions of $V$ ) into (14) to obtain the modal field. Concretely, the modal field is given by 


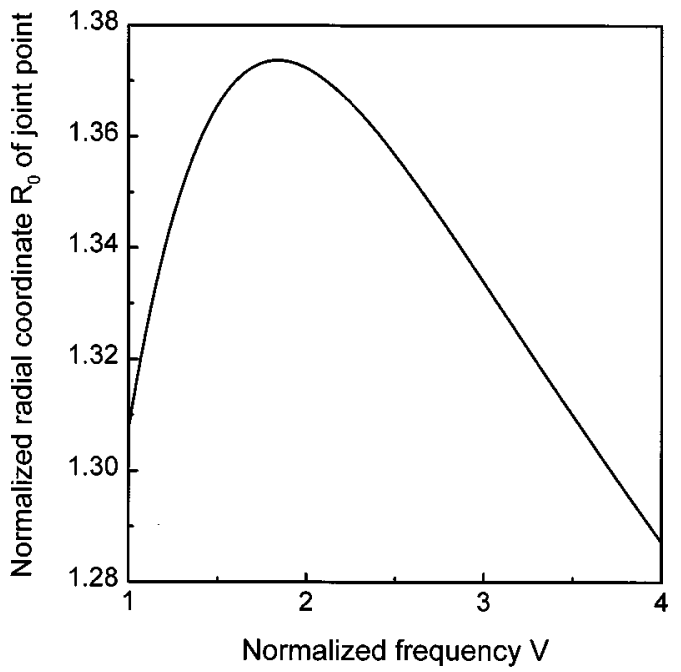

Fig. 3. Functional relation between the normalized radial coordinate $R_{0}(V)$ of the joint point and the normalized frequency $V$ : The Gaussian profile fiber.

(17), shown at the bottom of the next page, where $R_{0}$ is given by (16), $a_{1}, a_{3}, a_{5}, a_{7}, a_{9}$, and $W$ are given in the first paragraph of this subsection. The exact modal field, the approximate analytical formula presented here and the Gaussian approximation $\phi(R)=B \exp \left[-(\ln V) R^{2}\right]$ for $V=1.5$ and $V=2.5$ are shown in Fig. 2, where the field distributions have been normalized according to $\int_{0}^{\infty} \phi^{2} R d R=1, B$ is the normalized constant. From Fig. 2 one can see that our expression is far more accurate than the Gaussian approximation and still valid for small $V$ values. In order to test the accuracy of (15), we also evaluate the $U$ value corresponding to $V=1.5$ by employing (15). In this case, the exact value is $U=1.3169$; the $U$ value determined by the Gaussian approximation is $U=1.3457$; and the $U$ value determined by (15) is $U=1.3172$. From thses results, one can find that, just as we predict in Section II, the $U$ value determined by (15) is more accurate than that determined by the Gaussian approximation.

\section{B. The Gaussian Profile Fiber}

For a Gaussian profile fiber whose profile function has the form $f(R)=1-\exp \left(-R^{2}\right)$, the expansion coefficients $b_{m}$ are determined to be $b_{m}=(-1)^{(m / 2)-1} /(m / 2)$ ! for all even $m$ and $b_{m}=0$ for all odd $m$. Substituting $b_{m}$ and the relation $U^{2}=$ $2 V-1$, which is obtained from the Gaussian approximation [13], [14], into (8)-(10) and letting $m_{0}=9$, one can obtain $a_{2}=a_{4}=a_{6}=a_{8}=0, a_{1}=(2 V-1) / 2, a_{3}=-(4 V-$ 1) $/ 16, a_{5}=(6 V-1) / 96, a_{7}=\left(16 V^{2}-88 V+11\right) / 6144$

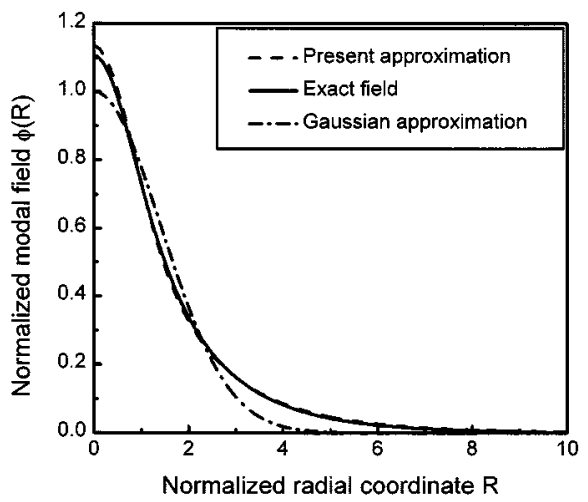

(a)

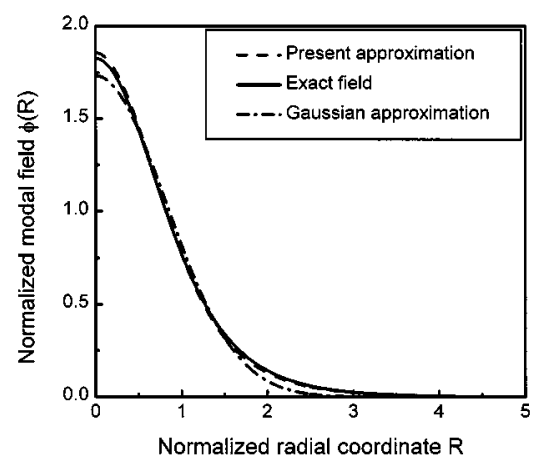

(b)

Fig. 4. Comparison among our approximate formula, the Gaussian approximation and the exact solution: The Gaussian profile fiber: (a) $V=1.5$ and (b) $V=2.5$.

and $a_{9}=\left(32 V^{3}-128 V^{2}+190 V-19\right) / 61440$. Substituting these coefficients $a_{m}$ and the relations $U^{2}=2 V-1$ and $W=$ $\sqrt{V^{2}-U^{2}}=V-1$ into (13), one can determine the joint point $R_{0}$. The change of $R_{0}$ with the variable $V$ is shown in Fig. 3. The functional relation $R_{0}(V)$ can be well fitted by the approximate expression

$$
R_{0}(V)=\left\{\begin{array}{lc}
-0.10096 V^{2}+0.36884 V+1.03953 \\
& 1 \leq V \leq 2, \\
-0.042575 V+1.45752, & 2<V \leq 4 .
\end{array}\right.
$$

The error of (18) with the exact value is less $0.25 \%$ in the range of $1 \leq V \leq 2$ and less than $0.45 \%$ in the range of $2<V \leq 4$. From (18) one can find that $R_{0}(V)$ is a approximate parabolic function in the range of $1 \leq V \leq 2$ and is a approximate linear function in the range of $2<V \leq 4$. From Fig. 3 one can further find that the total change of $R_{0}$ in the range of $1 \leq$

$$
\phi(R)= \begin{cases}A \exp \left[-\sum_{m=1}^{5} a_{2 m-1} R^{2 m} /(2 m)\right], & 0 \leq R \leq R_{0} \\ A \sqrt{R_{0} / R} \exp \left[-W\left(R-R_{0}\right)-\sum_{m=1}^{5} a_{2 m-1} R_{0}^{2 m} /(2 m)\right], & R \geq R_{0}\end{cases}
$$




$$
\phi(R)= \begin{cases}A \exp \left[-\sum_{m=1}^{5} a_{2 m-1} R^{2 m} /(2 m)\right], & 0 \leq R \leq R_{0} \\ A \sqrt{R_{0} / R} \exp \left[-W\left(R-R_{0}\right)-\sum_{m=1}^{5} a_{2 m-1} R_{0}^{2 m} /(2 m)\right], & R \geq R_{0}\end{cases}
$$

$V \leq 4$ is very small and the central point is about $R_{0}=1.34$. Similarly to the step profile fiber, the fundamental-mode field of a Gaussian profile fiber can be expressed as the closed form (19), shown at the top of the page, where $R_{0}(V)$ is given by (18), and $a_{1}, a_{3}, a_{5}, a_{7}, a_{9}$, and $W$ are given in the first paragraph of this subsection.

The exact modal field (we use the numerical method presented by [15] to obtain the exact numerical solutions), the approximate analytical formula presented here and the Gaussian approximation $\phi=C \exp \left[-(V-1) R^{2} / 2\right]$ for $V=1.5$ and $V=2.5$ are shown in Fig. 4, where the field distributions have been normalized according to $\int_{0}^{\infty} \phi^{2} R d R=1, C$ is the normalized constant. Again, the high accuracies of our approximate analytical description are observed. We also evaluate the $U$ value corresponding to $V=1.5$ by employing (15). In this case, the exact value is $U=1.3929$; the $U$ value determined by the Gaussian approximation is $U=1.4142$; and the $U$ value determined by (15) is $U=1.3932$. Again, we find that the $U$ value determined by (15) is more accurate than that determined by the Gaussian approximation.

\section{CONCLUSION}

We have presented an approximate analytical description for fundamental-mode fields of graded-index fibers by use of the power-series expansion method, the maximum-value condition $P(0)=0$ of the fundamantal-mode field $\phi(R)=\exp \left[-\int_{0}^{R} P(R) d R\right]$ at the $R=0$ point, and the decay law $P(R) \approx W+1 /(2 R)$ at large $R$. This new analytical description is much more accurate than the Gaussian approximation and at the same time keep the simplicity of the latter. As two special examples, we have presented the analytical approximate formulas for the fundamental-mode fields of a step profile fiber and a Gaussian profile fiber, and found that they are both highly accurate in the single-mode range by comparing them with the exact solutions. The results obtained in this paper can be used to conveniently evaluate the relevant parameters of a single-mode graded-index fiber.

\section{ACKNOWLEDGMENT}

The authors are indebted to the reviewers for their comments and proposals for improving the paper.

\section{REFERENCES}

[1] A. W. Snyder, "Asymptotic expressions for eigenfunctions and eigenvalues of a dielectric or optical waveguide," IEEE Trans. Microwave Theory Tech., vol. 17, pp. 1130-1138, 1969.

[2] D. Gloge, "Weakly guiding fibers," Appl. Opt., vol. 10, pp. 2252-2258, 1971.
[3] R. Yamada, T. Meiri, and N. Okamoto, "Guided waves along an optical fiber with parabolic index profile," J. Opt. Soc. Amer, vol. 67, pp. 96-103, 1977.

[4] W. A. Gambling, D. N. Payne, and N. Matsumura, "Cut-off frequency in radially inhomogeneous single-mode fiber," Electron. Lett., vol. 13, pp. 139-140, 1977.

[5] J. D. Love, "Power series solutions of the scalar wave equation for cladded, power-law profiles of arbitrary exponent," Opt. Quantum Electron., vol. 11, pp. 464-466, 1979.

[6] D. Marcuse, Light Transmission Optics, 2nd ed. Malabar, FL: Krieger, 1989, ch. 7.

[7] — "Gaussian approximation of the fundamental modes of gradedindex fibers," J. Opt. Soc. Amer, vol. 68, pp. 103-109, 1978.

[8] A. Sharma and A. K. Ghatak, "A variational analysis of single mode graded-index fibers," Opt. Commun., vol. 36, pp. 22-24, 1981.

[9] A. Sharma, S. I. Hosain, and A. K. Ghatak, "The fundamental mode of graded-index fibers: Simple and accurate variational methods," Opt. Quantum Electron., vol. 14, pp. 7-15, 1982.

[10] A. Ankiewicz and G. D. Peng, "Generalized gaussian approximation for single-mode fibers," J. Lightwave Technol., vol. 10, pp. 22-27, 1992.

[11] S. C. Chao, W. H. Tsai, and M. S. Wu, "Extended gaussian approximation for single-mode graded-index fibers," J. Lightwave Technol., vol. 12, pp. 392-395, 1994.

[12] G. De Angelis, G. Panariello, and A. Scaglione, "A variational method to approximate the field of weakly guiding optical fibers by Laguerre-Gauss/Bessel expansion,” J. Lightwave Technol., vol. 17, pp. 2665-2674, 1999

[13] A. W. Snyder and J. D. Love, Optical Waveguide Theory. New York: Chapman \& Hall, 1983, ch. 15.

[14] A. W. Snyder, "Understanding monomode optical fibers," Proc. IEEE, vol. 69 , pp. 6-13, 1981

[15] R. S. Anderssen, F. R. de Hoog, and J. D. Love, "A numerical technique for solving the scalar wave equation for gaussian and smoothed-out profiles," Opt. Quantum Electron., vol. 13, pp. 217-224, 1981.

[16] Y. Ohtera, O. Hanaizumi, and S. Kawakami, "Numerical analysis of eigenmodes and splice losses of thermally diffused expanded core fibers," J. Lightwave Technol., vol. 17, pp. 2675-2682, 1999.

[17] S. Schiff, Quantum Mechanics, 3rd ed. New York: McGraw-Hill, 1968 , ch. 8.

[18] E. K. Sharma, A. Sharma, and I. C. Goyal, "Propagation characteristics of single mode optical fibers with arbitrary index profiles: A simple numerical approach," IEEE Trans. Microwave Theory Tech., vol. MTT-30, pp. 1472-1477, 1982.

[19] W. A. Gambling and H. Matsumura, "Propagation in radially-inhomogeneous single-mode fiber," Opt. Quantum Electron., vol. 10, pp. 31-40, 1978.

Qing Cao, photograph and biography not available at the time of publication.

Sien Chi, photograph and biography not available at the time of publication. 